doi: $10.2306 /$ scienceasia1513-1874.2021.040

\title{
Some generalizations of numerical radius inequalities for Hilbert space operators
}

\author{
Chaojun Yang
}

Department of Mathematics, Nanjing University of Aeronautics and Astronautics, Nanjing 210016 China

e-mail: cjyangmath@163.com

Received 2 Dec 2020

Accepted 16 Mar 2021

ABSTRACT: In this article, we generalize several upper and lower bounds of the numerical radius inequalities for Hilbert space operators. In particular, we show that if $A \in B(\mathscr{H})$ with the Cartesian decomposition $A=B+i C$ and $f$ is an increasing concave function, then $f(\omega(A)) \geqslant \frac{1}{2}\|f(|B+C|)+f(|B-C|)\|$. This is a complementary result of El-Haddad and Kittaneh [Studia Math 182 (2007):133-140].

KEYWORDS: numerical radius, function, operator norm, Cartesian decomposition

MSC2010: 47A63 47A30

\section{INTRODUCTION}

Let $B(\mathscr{H})$ denote the $C^{*}$-algebra of all bounded linear operators on a complex Hilbert space $\mathscr{H}$ with an inner product $\langle\cdot\rangle$. For $A \in B(\mathscr{H})$, let $\|A\|$ denote the usual operator norm of $A$. The numerical range of $A$ is defined by $W(A)=\{\langle A x, x\rangle: x \in \mathscr{H},\|x\|=1\}$. The numerical radius of $A$ is defined by $\omega(A)=$ $\sup \{|\lambda|: \lambda \in W(A)\}$. We note that if $A \in B(\mathscr{H})$ and if $f$ is a non-negative increasing function on $[0, \infty)$, then $\|f(|A|)\|=f(\|A\|)$. Recall that $A \in B(\mathscr{H})$ is said to be hyponormal if $A^{*} A-A A^{*} \geqslant 0$, or equivalently if $\left\|A^{*} x\right\| \leqslant\|A x\|$.

It is well known that $\omega(\cdot)$ defines a norm on $B(\mathscr{H})$. In fact, for any $A \in B(\mathscr{H})$,

$$
\frac{1}{2}\|A\| \leqslant \omega(A) \leqslant\|A\|,
$$

which indicates the usual operator norm and the numerical radius norm are equivalent. For more information about numerical radius inequalities, readers are referred to $[1,2]$.

Before proceeding, we give the definition of geometrical convexity. First we note that all functions in this article satisfy the following condition unless otherwise specified: $J$ is a subinterval of $(0, \infty)$ and $f: J \rightarrow(0, \infty)$. We say that $f$ is geometrically convex if $f\left(a^{1-t} b^{t}\right) \leqslant f^{1-t}(a) f^{t}(b)$ for all $t \in[0,1]$. Recent studies on numerical radius inequalities involving convex and concave functions can be found in [3].

For positive real numbers $a$ and $b$, the classical Young inequality says that if $p, q>1$ such that $\frac{1}{p}+$ $\frac{1}{q}=1$, then

$$
a b \leqslant \frac{a^{p}}{p}+\frac{b^{q}}{q} .
$$

In particular, when $p=q=2$, this is the scalar arithmetic-geometric mean inequality.

A refinement of the scalar arithmetic-geometric mean inequality is presented in [4] as follows:

$$
\left(1+\frac{(\ln a-\ln b)^{2}}{8}\right) \sqrt{a b} \leqslant \frac{a+b}{2} .
$$

Kittaneh $[5,6]$ had shown the following inequalities which improved the inequalities in (1) by using several norm inequalities and ingenious techniques:

$$
\omega(A) \leqslant \frac{1}{2}\left(\|A\|+\left\|A^{2}\right\|^{1 / 2}\right),
$$

and

$$
\frac{1}{4}\left\||A|^{2}+\left|A^{*}\right|^{2}\right\| \leqslant \omega^{2}(A) \leqslant \frac{1}{2}\left\||A|^{2}+\left|A^{*}\right|^{2}\right\| .
$$

In [3], Omidvar et al presented the following inequalities which are improvements and generalizations of (3) and (4) for hyponormal operators, respectively. Let $A \in B(\mathscr{H})$ be a hyponormal operator, then for all $1 \leqslant r \leqslant 2$,

$$
\omega^{r}(A) \leqslant \frac{1}{2\left(1+\frac{\xi_{|A|}^{2}}{8}\right)^{r}}\left\||A|^{r}+\left|A^{*}\right|^{r}\right\|,
$$

and

$$
\omega^{r}(A) \leqslant \frac{1}{2\left(1+\frac{\xi_{|A|}^{2}}{8}\right)^{r}}\left(\|A\|^{r}+\left\||A|^{\frac{r}{2}}\left|A^{*}\right|^{\frac{r}{2}}\right\|\right),
$$


where $\xi_{|A|}^{2}=\inf _{\|x\|=1}\left\{\frac{\left\langle\left(|A|-\left|A^{*}\right|\right) x, x\right\rangle}{\left\langle\left(|A|+\left|A^{*}\right|\right) x, x\right\rangle}\right\}$.

In [7], Burqan and Abu-Rahma proved that if $A, B, C \in B(\mathscr{H})$ and $\left[\begin{array}{cc}A & B^{*} \\ B & C\end{array}\right] \geqslant 0$, then

$$
\omega^{r}(B) \leqslant \frac{1}{2}\left\|A^{r}+C^{r}\right\| \quad \text { for } \quad r \geqslant 1
$$

which gave an estimate for the numerical radius of the off-diagonal block operator matrices. For more information about numerical radius inequalities for block operator matrices and off-diagonal operator matrices, readers are referred to $[8,9]$. In the same paper, they also obtained a generalization of inequality (4) for two matrices as follows. Let $A, B \in$ $B(\mathscr{H})$ and $0<\alpha<1$, then, for $r \geqslant 1$,

$$
\begin{aligned}
\omega^{r}(A+B) \leqslant & \frac{1}{2} \|\left(\left|A^{*}\right|^{2 \alpha}+\left|B^{*}\right|^{2 \alpha}\right)^{r} \\
& \left.+\left(|A|^{2(1-\alpha)}+|B|^{2(1-\alpha}\right)\right)^{r} \| .
\end{aligned}
$$

In [10], El-Haddad and Kittaneh gave generalizations of inequalities (3) and (4) as follows. Let $A \in B(\mathscr{H})$ with the Cartesian decomposition $A=$ $B+i C$ and let $0<r \leqslant 2$. Then

$$
\omega^{r}(A) \leqslant\left\||B|^{r}+|C|^{r}\right\|
$$

They also showed that if $r \geqslant 2$, then

$$
\omega^{r}(A) \leqslant 2^{r / 2-1}|||B|^{r}+|C|^{r} \|
$$

and

$$
\begin{aligned}
2^{-r / 2-1} & \left\||B+C|^{r}+|B-C|^{r}\right\| \\
& \leqslant \omega^{r}(A) \leqslant \frac{1}{2}\left\||B+C|^{r}+|B-C|^{r}\right\| .
\end{aligned}
$$

In this paper, we first give a different proof of inequality (5) for $r \geqslant 2$, then we give some generalizations of several upper and lower bounds of the numerical radius inequalities for Hilbert space operators involving inequalities (6)-(10) for geometrically convex functions and concave functions.

\section{MAIN RESULTS}

We begin this section with some lemmas which will be necessary to prove our main results.

Lemma 1 ([11]) If $A \in B(\mathscr{H})$, then

$$
|\langle A x, y\rangle| \leqslant|\langle|A| x, y\rangle|^{\frac{1}{2}}\left|\left\langle\left|A^{*}\right| x, y\right\rangle\right|^{\frac{1}{2}}
$$

for all $x, y \in \mathscr{H}$.
Lemma 2 ([12]) If $A \in B(\mathscr{H})$ and $f$ and $g$ be nonnegative continuous functions on $[0, \infty)$ such that $f(t) g(t)=t$ for all $t \in[0, \infty)$, then

$$
|\langle A x, y\rangle|\|f(|A|) x\|\left\|g\left(\left|A^{*}\right|\right) y\right\|
$$

for all $x, y \in \mathscr{H}$.

Lemma 3 (McCarthy inequality [12]) Let $A$ be a positive operator in $B(\mathscr{H})$. For every unit vector $x \in \mathscr{H}$ and a given positive real number $r$,

(a) $\langle A x, x\rangle^{r} \leqslant\left\langle A^{r} x, x\right\rangle$ for $r \geqslant 1$,

(b) $\left\langle A^{r} x, x\right\rangle \leqslant\langle A x, x\rangle^{r}$ for $0<r \leqslant 1$.

Lemma 4 ([3]) For each $\alpha \geqslant 1$, we have

$$
\frac{\alpha-1}{\alpha+1} \leqslant \ln \alpha .
$$

Lemma 5 Let $A \in B(\mathscr{H})$ be a hyponormal operator and let $f$ and $g$ be nonnegative continuous functions on $[0, \infty)$ such that $f(t) g(t)=t$ for all $t \in[0, \infty)$. Then

$$
\begin{aligned}
\omega^{r}(A) \leqslant \frac{1}{2\left(1+\frac{\xi_{|A|}^{2}}{8}\right)^{r}} & \| \frac{1}{p}\left(f^{2 p}\left(|A|^{\frac{r}{2}}\right)+f^{2 p}\left(\left|A^{*}\right|^{\frac{r}{2}}\right)\right) \\
& +\frac{1}{q}\left(g^{2 q}\left(|A|^{\frac{r}{2}}\right)+g^{2 q}\left(\left|A^{*}\right|^{\frac{r}{2}}\right)\right) \|,
\end{aligned}
$$

where $r \geqslant 2, p, q \geqslant 1$ such that $\frac{1}{p}+\frac{1}{q}=1$ and $\xi_{|A|}=$ $\inf _{\|x\|=1}\left\{\frac{\left\langle\left(|A|-\left|A^{*}\right|\right) x, x\right\rangle}{\left\langle\left(|A|+\left|A^{*}\right|\right) x, x\right\rangle}\right\}$.

Proof: Since $A$ is a hyponormal operator we have $1 \leqslant \frac{\langle|A| x, x\rangle}{\left\langle\left|A^{*}\right| x, x\right\rangle}$ for each $x \in \mathscr{H}$. On choosing $\alpha=\frac{\langle|A| x, x\rangle}{\left\langle\left|A^{*}\right| x, x\right\rangle}$ in Lemma 4 we get

$$
0 \leqslant \frac{\left\langle\left(|A|-\left|A^{*}\right|\right) x, x\right\rangle}{\left\langle\left(|A|+\left|A^{*}\right|\right) x, x\right\rangle} \leqslant \ln \frac{\langle|A| x, x\rangle}{\left\langle\left|A^{*}\right| x, x\right\rangle} .
$$

Whence

$$
\inf _{\|x\|=1} \frac{\left\langle\left(|A|-\left|A^{*}\right|\right) x, x\right\rangle}{\left\langle\left(|A|+\left|A^{*}\right|\right) x, x\right\rangle} \leqslant \ln \frac{\langle|A| x, x\rangle}{\left\langle\left|A^{*}\right| x, x\right\rangle} .
$$

We denote the expression on the left side of (11) by $\xi_{|A|}$. In inequality (2), by taking $a=\langle|A| x, x\rangle$ and $b=\left\langle\left|A^{*}\right| x, x\right\rangle$ and taking into account that $\xi_{|A|} \leqslant$ $\ln \frac{\langle|A| x, x\rangle}{\left\langle\left|A^{*}\right| x, x\right\rangle}$, we infer that

$$
\sqrt{\langle|A| x, x\rangle\left\langle\left|A^{*}\right| x, x\right\rangle} \leqslant \frac{1}{2\left(1+\frac{\xi_{|A|}^{2}}{8}\right)}\left\langle\left(|A|+\left|A^{*}\right|\right) x, x\right\rangle .
$$


By Lemma 1, we get

$$
|\langle A x, x\rangle| \leqslant \frac{1}{2\left(1+\frac{\xi_{|A|}^{2}}{8}\right)}\left\langle\left(|A|+\left|A^{*}\right|\right) x, x\right\rangle .
$$

Now by taking $\|x\|=1$, we have

$$
\begin{aligned}
&|\langle A x, x\rangle|^{r} \leqslant \frac{1}{2^{r}\left(1+\frac{\xi_{|A|}^{2}}{8}\right)^{r}}\left\langle\left(|A|+\left|A^{*}\right|\right) x, x\right\rangle^{r} \\
& \leqslant \frac{1}{2\left(1+\frac{\xi_{|A|}^{2}}{8}\right)^{r}}\left(\langle|A| x, x\rangle^{r}+\left\langle\left|A^{*}\right| x, x\right\rangle^{r}\right) \\
& \leqslant \frac{1}{2\left(1+\frac{\xi_{|A|}^{2}}{8}\right)^{r}}\left(\left\langle|A|^{\frac{r}{2}} x, x\right\rangle^{2}+\left\langle\left|A^{*}\right|^{\frac{r}{2}} x, x\right\rangle^{2}\right) \\
& \leqslant \frac{1}{2\left(1+\frac{\xi_{|A|}^{2}}{8}\right)^{r}}\left(\left\langle f^{2}\left(|A|^{\frac{r}{2}}\right) x, x\right\rangle\left\langle g^{2}\left(|A|^{\frac{r}{2}}\right) x, x\right\rangle\right. \\
&\left.+\left\langle f^{2}\left(\left|A^{*}\right|^{\frac{r}{2}}\right) x, x\right\rangle\left\langle g^{2}\left(\left|A^{*}\right|^{\frac{r}{2}}\right) x, x\right\rangle\right) \quad(\text { Lemma } 2) \\
& \leqslant \frac{1}{2\left(1+\frac{\xi_{|A|}^{2}}{8}\right)^{r}}\left(\frac{1}{p}\left\langle f^{2}\left(|A|^{\frac{r}{2}}\right) x, x\right\rangle^{p}+\frac{1}{q}\left\langle g^{2}\left(|A|^{\frac{r}{2}}\right) x, x\right\rangle^{q}\right. \\
&\left.+\frac{1}{p}\left\langle f^{2}\left(\left|A^{*}\right|^{\frac{r}{2}}\right) x, x\right\rangle^{p}+\frac{1}{q}\left\langle g^{2}\left(\left|A^{*}\right|^{\frac{r}{2}}\right) x, x\right\rangle^{q}\right) \\
& \leqslant \frac{1}{2\left(1+\frac{\xi_{|A|}^{2}}{8}\right)^{r}}\left(\frac{1}{p}\left\langle f^{2 p}\left(|A|^{\frac{r}{2}}\right) x, x\right\rangle+\frac{1}{q}\left\langle g^{2 q}\left(|A|^{\frac{r}{2}}\right) x, x\right\rangle\right. \\
&\left.+\frac{1}{p}\left\langle f^{2 p}\left(\left|A^{*}\right|^{\frac{r}{2}}\right) x, x\right\rangle+\frac{1}{q}\left\langle g^{2 q}\left(\left|A^{*}\right|^{\frac{r}{2}}\right) x, x\right\rangle\right) \\
&= \frac{1}{2\left(1+\frac{\xi_{|A|}^{2}}{8}\right)^{r}}\left(\frac{1}{p}\left(f^{2 p}\left(|A|^{\frac{r}{2}}\right)+f^{2 p}\left(\left|A^{*}\right|^{\frac{r}{2}}\right)\right)\right. \\
&\left.+\frac{1}{q}\left\langle\left(g^{2 q}\left(|A|^{\frac{r}{2}}\right)+g^{2 q}\left(\left|A^{*}\right|^{\frac{r}{2}}\right)\right)\right) x, x\right\rangle .
\end{aligned}
$$

Now the result follows by taking the supremum over all unit vectors in $\mathscr{H}$.

Theorem 1 Let $A \in B(\mathscr{H})$ is a hyponormal operator. Then, for all $r \geqslant 1$,

$$
\omega^{r}(A) \leqslant \frac{1}{2\left(1+\frac{\xi_{|A|}^{2}}{8}\right)^{r}}\left\||A|^{r}+\left|A^{*}\right|^{r}\right\|,
$$

where $\xi_{|A|}=\inf _{\|x\|=1}\left\{\frac{\left\langle\left(|A|-\left|A^{*}\right|\right) x, x\right\rangle}{\left\langle\left(|A|+\left|A^{*}\right|\right) x, x\right\rangle}\right\}$.

Proof: The case $1 \leqslant r \leqslant 2$ in Theorem 1 follows from the result of Omidvar et al. The case $r \geqslant 2$ is a direct result of Lemma 5 by setting $p=q=2$ and $f(t)=$ $g(t)=t^{\frac{1}{2}}$.

Theorem 2 Let $A \in B(\mathscr{H})$ is a hyponormal operator. Then, for all $r \geqslant 1$,

$$
\omega^{r}(A) \leqslant \frac{1}{2\left(1+\frac{\xi_{|A|}^{2}}{8}\right)^{r}}\left(\|A\|^{r}+\left\||A|^{\frac{r}{2}}\left|A^{*}\right|^{\frac{r}{2}}\right\|\right),
$$

where $\xi_{|A|}=\inf _{\|x\|=1}\left\{\frac{\left\langle\left(|A|-\left|A^{*}\right|\right) x, x\right\rangle}{\left\langle\left(|A|+\left|A^{*}\right|\right) x, x\right\rangle}\right\}$.

Proof: Straightforward.

Lemma 6 ([13]) Let $A, B, C \in B(\mathscr{H})$ such that $\left[\begin{array}{ll}A & B^{*} \\ B & C\end{array}\right] \geqslant 0$. Then $|\langle B x, y\rangle|^{2} \leqslant\langle A x, x\rangle\langle C y, y\rangle$ for all $x, y \in \mathscr{H}$.

Theorem 3 Let $A, B, C \in B(\mathscr{H})$ be such that $\left[\begin{array}{ll}A & B^{*} \\ B & C\end{array}\right] \geqslant 0$ and $f$ be an increasing geometrically convex function. If in addition $f$ is convex, then

$$
f(\omega(B)) \leqslant \frac{1}{2}\|f(A)+f(C)\| .
$$

Proof: For any unit vector $x \in \mathscr{H}$, we have the following chain of inequalities

$$
\begin{aligned}
f(|\langle B x, x\rangle|) & \leqslant f\left(\langle A x, x\rangle^{\frac{1}{2}}\langle C x, x\rangle^{\frac{1}{2}}\right) \quad \text { (Lemma 6) } \\
& \leqslant \sqrt{f(\langle A x, x\rangle) f(\langle C x, x\rangle)} \\
& \leqslant \sqrt{\langle f(A) x, x\rangle\langle f(C) x, x\rangle} \\
& \leqslant \frac{1}{2}\langle(f(A)+f(C)) x, x\rangle .
\end{aligned}
$$

Hence,

$$
\begin{aligned}
f(\omega(B)) & =f\left(\sup _{\|x\|=1}|\langle B x, x\rangle|\right) \\
& =\sup _{\|x\|=1} f(|\langle B x, x\rangle|) \\
& \leqslant \sup _{\|x\|=1} \frac{1}{2}\langle(f(A)+f(C)) x, x\rangle \\
& =\frac{1}{2}\|(f(A)+f(C))\|,
\end{aligned}
$$

as required.

Remark 1 It is easy to verify that the function $f(t)=t^{r}(r \geqslant 1)$ satisfies the assumptions of Theorem 3, thus (6) is a special case of Theorem 3.

Lemma 7 ([12]) Let $A, B, C \in B(\mathscr{H})$ such that $A$ and $B$ are positive, $B C=C A$, and let $f$ and $g$ be nonnegative functions on $[0, \infty)$ which are continuous and satisfying the relation $f(t) g(t)=t$ for all $t \in[0, \infty)$.

If $\left[\begin{array}{ll}A & B^{*} \\ B & C\end{array}\right] \geqslant 0$, then $\left[\begin{array}{cc}f^{2}(A) & B^{*} \\ B & g^{2}(C)\end{array}\right] \geqslant 0$.

Theorem 4 Let $A_{i}, B_{i}, X_{i} \in B(\mathscr{H})(i=1, \ldots, n)$, and let $f_{i}$ and $g_{i}(i=1, \ldots, n)$ be nonnegative functions on $[0, \infty)$ which are continuous and satisfying the 
relation $f_{i}(t) g_{i}(t)=t$ for all $t \in[0, \infty)$. Then for any positive integer $m$, it holds

$$
\begin{aligned}
\omega^{r}\left(\sum_{i=1}^{n} A_{i} X_{i}\left|X_{i}\right|^{m-1} B_{i}^{*}\right) \leqslant & \frac{1}{2} \|\left(\sum_{i=1}^{n} A_{i} f_{i}^{2}\left(\left|X_{i}^{*}\right|^{m}\right) A_{i}^{*}\right)^{r} \\
& +\left(\sum_{i=1}^{n} B_{i} g_{i}^{2}\left(\left|X_{i}\right|^{m}\right) B_{i}^{*}\right)^{r} \|,
\end{aligned}
$$

where $r \geqslant 1$.

Proof: Note that for any $X_{i} \in B(\mathscr{H})$ it admits a polar decomposition $X_{i}=U_{i}\left|X_{i}\right|$. Since an operator $A$ on $\mathscr{H}$ is positive if and only if the operator $\left[\begin{array}{cc}A & A \\ A & A\end{array}\right]$ on $\mathscr{H} \oplus \mathscr{H}$ is positive, by simple computations, we have

$$
\begin{aligned}
& {\left[\begin{array}{cc}
\left|X_{i}^{*}\right|^{m} & \left|X_{i}\right|^{m} U_{i}^{*} \\
U_{i}\left|X_{i}\right|^{m} & U_{i}\left|X_{i}\right|^{m} U_{i}^{*}
\end{array}\right]} \\
& \quad=\left[\begin{array}{cc}
U_{i} & 0 \\
0 & I
\end{array}\right]\left[\begin{array}{ll}
\left|X_{i}\right|^{m} & \left|X_{i}\right|^{m} \\
\left|X_{i}\right|^{m} & \left|X_{i}\right|^{m}
\end{array}\right]\left[\begin{array}{cc}
U_{i}^{*} & 0 \\
0 & I
\end{array}\right] \geqslant 0,
\end{aligned}
$$

which indicates

$$
\left[\begin{array}{cc}
\left|X_{i}^{*}\right|^{m} & U_{i}\left|X_{i}\right|^{m} \\
\left|X_{i}\right|^{m} U_{i}^{*} & \left|X_{i}^{*}\right|^{m}
\end{array}\right]=\left[\begin{array}{cc}
U_{i}\left|X_{i}\right|^{m} U_{i}^{*} & U_{i}\left|X_{i}\right|^{m} \\
\left|X_{i}\right|^{m} U_{i}^{*} & \left|X_{i}^{*}\right|^{m}
\end{array}\right] \geqslant 0 .
$$

Therefore

$$
\left[\begin{array}{cc}
\left|X_{i}^{*}\right|^{m} & X_{i}\left|X_{i}\right|^{m-1} \\
\left|X_{i}\right|^{m-1} X_{i}^{*} & \left|X_{i}\right|^{m}
\end{array}\right]=\left[\begin{array}{cc}
U_{i}\left|X_{i}\right|^{m} U_{i}^{*} & U_{i}\left|X_{i}\right|^{m} \\
\left|X_{i}\right|^{m} U_{i}^{*} & \left|X_{i}\right|^{m}
\end{array}\right] \geqslant 0 .
$$

For the special case $m=1$, we set $\left|X_{i}\right|^{0}=I$. To apply Lemma 7, note that $\left|X_{i}\right|^{m}\left|X_{i}\right|^{m-1} X_{i}^{*}=\left|X_{i}\right|^{2 m} U^{*}=$ $\left|X_{i}\right|^{m-1}\left|X_{i}\right| U^{*} U\left|X_{i}\right|^{m} U^{*}=\left|X_{i}\right|^{m-1} X_{i}^{*}\left|X_{i}^{*}\right|^{m}$. Thus $\left[\begin{array}{cc}f_{i}^{2}\left(\left|X_{i}^{*}\right|^{m}\right) & X_{i}\left|X_{i}\right|^{m-1} \\ \left|X_{i}\right|^{m-1} X_{i}^{*} & g_{i}^{2}\left(\left|X_{i}\right|^{m}\right)\end{array}\right] \geqslant 0$. Pre-post multiply the above matrix by $\left[\begin{array}{cc}A & 0 \\ 0 & B\end{array}\right]$ and $\left[\begin{array}{cc}A^{*} & 0 \\ 0 & B^{*}\end{array}\right]$, respectively, we have $\left[\begin{array}{ll}A f_{i}^{2}\left(\left|X_{i}^{*}\right|^{m}\right) A^{*} & A X_{i}\left|X_{i}\right|^{m-1} B^{*} \\ B\left|X_{i}\right|^{m-1} X_{i}^{*} A^{*} & B g_{i}^{2}\left(\left|X_{i}\right|^{m}\right) B^{*}\end{array}\right] \geqslant 0$. Summing up the previous matrices for $i=1,2, \ldots, n$, we have

$$
\left[\begin{array}{ll}
\sum_{i=1}^{n} A f_{i}^{2}\left(\left|X_{i}^{*}\right|^{m}\right) A^{*} & \sum_{i=1}^{n} A X_{i}\left|X_{i}\right|^{m-1} B^{*} \\
\sum_{i=1}^{n} B\left|X_{i}\right|^{m-1} X_{i}^{*} A^{*} & \sum_{i=1}^{n} B g_{i}^{2}\left(\left|X_{i}\right|^{m}\right) B^{*}
\end{array}\right] \geqslant 0 .
$$

By applying Theorem 3 to the above matrix and letting $f(t)=t^{r}(r \geqslant 1)$, we thus obtain the result.

Remark 2 In Theorem 4, if we take $m=n=1,0 \leqslant$ $\alpha \leqslant 1, f(t)=t^{\alpha}, g(t)=t^{1-\alpha}, A_{1}=B_{1}=I$, and $X_{1}=$ $A$, we get Theorem 1 in [10].
Remark 3 In Theorem 4, if we take $m=1, n=2$, $0 \leqslant \alpha \leqslant 1, f(t)=t^{\alpha}, g(t)=t^{1-\alpha}, A_{i}=B_{i}=I(i=$ $1,2), X_{1}=A$, and $X_{2}=B$, we get (7).

Remark 4 In Theorem 4, if we take $m=n=1,0 \leqslant$ $\alpha \leqslant 1, f(t)=t^{\alpha}, g(t)=t^{1-\alpha}, A_{1}=B^{*}, B_{1}=A$, and $X_{1}=I$, we get Theorem 1 in [14].

Theorem 5 Let $A \in B(\mathscr{H})$ with the Cartesian decomposition $A=B+i C$ and $f$ be an increasing concave function. Then

$$
f\left(\omega^{2}(A)\right) \leqslant\left\|f\left(|B|^{2}\right)+f\left(|C|^{2}\right)\right\| .
$$

Proof: Since $A=B+i C$ is the Cartesian decomposition of $A$, we have $|\langle A x, x\rangle|^{2}=\langle B x, x\rangle^{2}+\langle C x, x\rangle^{2}$ for every unit vector $x$. Therefore

$$
\begin{aligned}
f\left(|\langle A x, x\rangle|^{2}\right) & =f\left(\langle B x, x\rangle^{2}+\langle C x, x\rangle^{2}\right) \\
& \leqslant f\left(\langle|B| x, x\rangle^{2}+\langle|C| x, x\rangle^{2}\right) \\
& \leqslant f\left(\left\langle|B|^{2} x, x\right\rangle+\left\langle|C|^{2} x, x\right\rangle\right) \\
& =f\left(\left\langle\left(|B|^{2}+|C|^{2}\right) x, x\right\rangle\right) .
\end{aligned}
$$

Since $\|f(A+B)\| \leqslant\|f(A)+f(B)\|$ for positive operator $A, B$ and every nonnegative concave function $f$ on $[0, \infty)$, it follows that

$$
\begin{aligned}
f\left(\omega^{2}(A)\right) & =f\left(\sup _{\|x\|=1}|\langle A x, x\rangle|^{2}\right) \\
& =\sup _{\|x\|=1} f\left(|\langle A x, x\rangle|^{2}\right) \\
& \leqslant \sup _{\|x\|=1} f\left(\left\langle\left(|B|^{2}+|C|^{2}\right) x, x\right\rangle\right) \\
& =f\left(\left\||B|^{2}+|C|^{2}\right\|\right) \\
& =\left\|f\left(|B|^{2}+|C|^{2}\right)\right\| \\
& \leqslant\left\|f\left(|B|^{2}\right)+f\left(|C|^{2}\right)\right\|,
\end{aligned}
$$

completing the proof.

Remark 5 Since the function $f(t)=t^{r}(0<r \leqslant 1)$ satisfies the assumptions of Theorem 5 , it is clear that inequality (8) is a special case of Theorem 5.

Theorem 6 Let $A \in B(\mathscr{H})$ with the Cartesian decomposition $A=B+i C$ and $f$ be an increasing geometrically convex function. If in addition $f$ is convex and $f(1)=1$, then

$$
f\left(\frac{\omega(A)}{\sqrt{2}}\right) \leqslant \sqrt{\frac{\left\|f\left(|B|^{2}\right)+f\left(|C|^{2}\right)\right\|}{2}} .
$$


Proof: For every unit vector $x \in \mathscr{H}$, we have

$$
\begin{aligned}
f\left(\frac{|\langle A x, x\rangle|}{\sqrt{2}}\right) & =f\left(\left(\frac{\langle B x, x\rangle^{2}+\langle C x, x\rangle^{2}}{2}\right)^{\frac{1}{2}}\right) \\
& \leqslant f^{\frac{1}{2}\left(\frac{\langle B x, x\rangle^{2}+\langle C x, x\rangle^{2}}{2}\right) f^{\frac{1}{2}}(1)} \\
& \leqslant \sqrt{\frac{f\left(\langle B x, x\rangle^{2}\right)+f\left(\langle C x, x\rangle^{2}\right)}{2}} \\
& \leqslant \sqrt{\frac{f\left(\langle|B| x, x\rangle^{2}\right)+f\left(\langle|C| x, x\rangle^{2}\right)}{2}} \\
& \leqslant \sqrt{\frac{f\left(\left\langle|B|^{2} x, x\right\rangle\right)+f\left(\left\langle|C|^{2} x, x\right\rangle\right)}{2}} \\
& \leqslant \sqrt{\frac{\left\langle f\left(|B|^{2}\right) x, x\right\rangle+\left\langle f\left(|C|^{2}\right) x, x\right\rangle}{2}} \\
& =\sqrt{\frac{\left\langle\left(f\left(|B|^{2}\right)+f\left(|C|^{2}\right)\right) x, x\right\rangle}{2}} .
\end{aligned}
$$

Hence

$$
\begin{aligned}
f\left(\frac{\omega(A)}{\sqrt{2}}\right) & =f\left(\sup _{\|x\|=1} \frac{|\langle A x, x\rangle|}{\sqrt{2}}\right) \\
& =\sup _{\|x\|=1} f\left(\frac{|\langle A x, x\rangle|}{\sqrt{2}}\right) \\
& \leqslant \sup _{\|x\|=1} \sqrt{\frac{\left\langle\left(f\left(|B|^{2}\right)+f\left(|C|^{2}\right)\right) x, x\right\rangle}{2}} \\
& =\sqrt{\frac{\sup _{\|x\|=1}\left\langle\left(f\left(|B|^{2}\right)+f\left(|C|^{2}\right)\right) x, x\right\rangle}{2}} \\
& =\sqrt{\frac{\left\|f\left(|B|^{2}\right)+f\left(|C|^{2}\right)\right\|}{2}},
\end{aligned}
$$

as required.

Remark 6 Since the function $f(t)=t^{r}(r \geqslant 1)$ satisfies the assumptions of Theorem 6 , it is clear that inequality (9) is a special case of Theorem 6.

Theorem 7 Let $A \in B(\mathscr{H})$ with the Cartesian decomposition $A=B+i C$ and $f$ be an increasing geometrically convex function. If in addition $f$ is convex and $f(1)=1$, then

$$
f(\omega(A)) \leqslant \sqrt{\frac{\left\|f\left(|B+C|^{2}\right)+f\left(|B-C|^{2}\right)\right\|}{2}} .
$$

Proof: Since for any two real numbers $a$ and $b$, we have $a^{2}+b^{2}=\frac{(a+b)^{2}+(a-b)^{2}}{2}$. It follows that

$$
\begin{aligned}
& f(|\langle A x, x\rangle|)=f\left(\left(\langle B x, x\rangle^{2}+\langle C x, x\rangle^{2}\right)^{\frac{1}{2}}\right) \\
& \quad=f\left(\left(\frac{\langle(B+C) x, x\rangle^{2}+\langle(B-C) x, x\rangle^{2}}{2}\right)^{\frac{1}{2}}\right)
\end{aligned}
$$

for any unit vector $x$, the rest of the proof follows from Theorem 6.

Remark 7 Since the function $f(t)=t^{r}(r \geqslant 1)$ satisfies the assumptions of Theorem 7 , it is clear that the right-hand side of inequality (10) is a special case of Theorem 7.

Theorem 8 Let $A \in B(\mathscr{H})$ with the Cartesian decomposition $A=B+i C$ and $f$ be an increasing concave function. Then

$$
f(\omega(A)) \geqslant \frac{1}{2}\|f(|B+C|)+f(|B-C|)\| .
$$

Proof: Since for any two real numbers $a$ and $b$, we have $a^{2}+b^{2}=\frac{(a+b)^{2}+(a-b)^{2}}{2}$. It follows that

$$
\begin{aligned}
f(|\langle A x, x\rangle|) & =f\left(\left(\langle B x, x\rangle^{2}+\langle C x, x\rangle^{2}\right)^{\frac{1}{2}}\right) \\
= & f\left(\left(\frac{\langle(B+C) x, x\rangle^{2}+\langle(B-C) x, x\rangle^{2}}{2}\right)^{\frac{1}{2}}\right) \\
\geqslant & f\left(\frac{|\langle(B+C) x, x\rangle|+|\langle(B-C) x, x\rangle|}{2}\right) \\
\geqslant & \frac{f(|\langle(B+C) x, x\rangle|)+f(|\langle(B-C) x, x\rangle|)}{2} .
\end{aligned}
$$

By taking the supremum over unit vector $x$, we obtain

$$
f(\omega(A)) \geqslant \frac{f(\|(B+C)\|)+f(\|(B-C)\|)}{2} .
$$

Thus by the triangle inequality for operator norm, we have

$$
\begin{aligned}
f(\omega(A)) & \geqslant \frac{f(\|(B+C)\|)+f(\|(B-C)\|)}{2} \\
& =\frac{\|f(|B+C|)\|+\|f(|B-C|)\|}{2} \\
& \geqslant \frac{\|f(|B+C|)+f(|B-C|)\|}{2},
\end{aligned}
$$

which completes the proof.

Remark 8 Since the function $f(t)=t^{r}(0<r \leqslant 1)$ satisfies the assumptions of Theorem 8 , we have $\omega^{r}(A) \geqslant \frac{1}{2}\left\||B+C|^{r}+|B-C|^{r}\right\|$ for $0<r \leqslant 1$, which can be viewed as a complement of the left-hand side part of inequality (10). To show that $\omega(A) \geqslant$ $\frac{1}{2}\||B+C|+|B-C|\|$ is sharp, consider $A=\left[\begin{array}{ll}1 & 0 \\ 0 & 1\end{array}\right]$, then $\omega(A)=1$ and $\||B+C|+|B-C|\|=2$.

Theorem 9 Let $A \in B(\mathscr{H})$ with the Cartesian decomposition $A=B+i C$ and $f$ be an increasing concave function. Then

$$
f\left(\frac{\omega(A)}{\sqrt{2}}\right) \geqslant \frac{1}{2}\|f(|B|)+f(|C|)\| .
$$


Proof: For every unit vector $x \in \mathscr{H}$, we have

$$
\begin{aligned}
f\left(\frac{|\langle A x, x\rangle|}{\sqrt{2}}\right) & =f\left(\left(\frac{\langle B x, x\rangle^{2}+\langle C x, x\rangle^{2}}{2}\right)^{\frac{1}{2}}\right) \\
& \geqslant f\left(\frac{|\langle B x, x\rangle|+|\langle C x, x\rangle|}{2}\right) \\
& \geqslant \frac{f(|\langle B x, x\rangle|)+f(|\langle C x, x\rangle|)}{2} .
\end{aligned}
$$

By taking the supremum over $x$, we obtain

$$
f\left(\frac{\omega(A)}{\sqrt{2}}\right) \geqslant \frac{f(\|B\|)+f(\|C\|)}{2} .
$$

Thus

$$
\begin{aligned}
f\left(\frac{\omega(A)}{\sqrt{2}}\right) & \geqslant \frac{f(\|B\|)+f(\|C\|)}{2} \\
& =\frac{\|f(|B|)\|+\|f(|C|)\|}{2} \\
& \geqslant \frac{\|f(|B|)+f(|C|)\|}{2},
\end{aligned}
$$

which completes the proof.

Remark 9 Since the function $f(t)=t^{r}(0<r \leqslant 1)$ satisfies the assumptions of Theorem 9 , we have $\omega^{r}(A) \geqslant 2^{\frac{r}{2}-1}|||B|^{r}+|C|^{r} \|$ for $0<r \leqslant 1$, which can be viewed as a complement and reverse of inequalities (8) and (9). To show that $\omega(A) \geqslant \frac{1}{\sqrt{2}} \||B|+|C|||$ is

sharp, consider $A=(1+i)\left[\begin{array}{ll}1 & 0 \\ 0 & 1\end{array}\right]$, then $\omega(A)=\sqrt{2}$ and $\||B|+|C|||=2$.

Acknowledgements: This project was funded by China Postdoctoral Science Foundation (No. 2020M681575).

\section{REFERENCES}

1. Dragomir SS (2013) Inequalities for the Numerical Radius of Linear Operators in Hilbert Spaces, Springer Briefs in Mathematics, Springer, Cham.
2. Kittaneh F, Moslehian MS, Yamazaki T (2015) Cartesian decomposition and numerical radius inequalities. Linear Algebra Appl 471, 46-53.

3. Omidvar ME, Moradi HR, Shebrawi K (2018) Sharpening some classical numerical radius inequalities. Oper Matrices 12, 407-416.

4. Zou L, Jiang Y (2015) Improved arithmeticgeometric mean inequality and its application. $J$ Math Inequal 9, 107-111.

5. Kittaneh F (2003) A numerical radius inequality and an estimate for the numerical radius of the Frobenius companion matrix. Studia Math 158, 11-17.

6. Kittaneh F (2005) Numerical radius inequalities for Hilbert space operators. Studia Math 168, 73-80.

7. Burqan A, Abu-Rahma A (2019) Generalizations of numerical radius inequalities related to block matrices. Filomat 33, 4981-4987.

8. Bakherad M, Shebrawi K (2018) Upper bounds for numerical radius inequalities involving off-diagonal operator matrices. Ann Funct Anal 9, 297-309.

9. Sahoo S, Das N, Mishra D (2019) Numerical radius inequalities for operator matrices. Adv Oper Theory 4, 197-214.

10. El-Haddad M, Kittaneh F (2007) Numerical radius inequalities for Hilbert space operators, II. Studia Math 182, 133-140.

11. Halmos PR (1982) A Hilbert Space Problem Book, 2nd edn, Springer, New York.

12. Kittaneh F (1988) Notes on some inequalities for Hilbert space operators. Publ Res Inst Math Sci 24, 276-293.

13. Moslehian MS, Kian M, Xu Q (2019) Positivity of $2 \times 2$ block matrices of operators. Banach J Math Anal 13, 726-743.

14. Dragomir SS (2009) Power inequalities for the numerical radius of a product of two operators in Hilbert spaces. Sarajevo J Math 5, 269-278. 